BMJ Open Sport \& Exercise Medicine

\title{
Intermittent fasting: eating by the clock for health and exercise performance
}

Sumona Mandal (D) , Niall Simmons, ${ }^{1}$ Sidra Awan, ${ }^{2}$ Karim Chamari, ${ }^{3}$
Irfan Ahmed (iD ${ }^{4}$

To cite: Mandal S, Simmons N, Awan S, et al. Intermittent fasting: eating by the clock for health and exercise performance. BMJ Open Sport \& Exercise Medicine 2022;8:e001206. doi:10.1136/ bmjsem-2021-001206

\section{Check for updates}

\section{(c) Author(s) (or their} employer(s)) 2022. Re-use permitted under CC BY-NC. No commercial re-use. See rights and permissions. Published by BMJ.

${ }^{1}$ University College London, Medical School, London, UK ${ }^{2}$ University College London Hospitals NHS Foundation Trust, London, UK

${ }^{3}$ Aspetar, Orthopaedic and Sports Medicine Hospital, Doha Qatar

${ }^{4}$ Sports Medicine Department, Homerton University Hospital, London, UK

Correspondence to Dr Irfan Ahmed; irfan.ahmed5@nhs.net

\section{ABSTRACT}

Intermittent fasting (IF) is an increasingly popular dietary practice, and its implementation is found throughout human civilisation in various cultural, spiritual and religious traditions. Emerging evidence has shown that the health benefits of IF stretch beyond calorie restriction and weight loss. These benefits include metabolic shifts in energy production, the optimisation of peripheral circadian clocks, and overall improvement in physiological markers of metabolic health. IF has been proposed to reduce systemic inflammation and have a role in the prevention and treatment of chronic disease. For the athlete, IF protocols offer a potential new frontier for maintaining performance in the fasted state. They may allow athletes to optimise training adaptions, while respecting individual cultural, religious, and/or spiritual preferences to fast and exercise. Below, we discuss the physiological impact of fasted exercise while highlighting areas for future work to improve our understanding and implementation of the practice for the benefit of both the active general community and sporting populations.

\section{INTRODUCTION}

Intermittent fasting (IF) is a popular dietary practice, which consists of regular alternating periods of unrestricted dietary consumption and abstinence from caloric intake. Emerging evidence shows IF has short and long-term benefits for health and exercise performance that go beyond facilitating weight-loss. ${ }^{1-4}$ Despite its widespread use, there remains inconclusive evidence on how best to use IF for health and well-being. We discuss the emerging evidence related to IF and the potential for its use in personalised medicine.

\section{What is IF?}

IF practices have been commonly performed across both ancient and modern societies for religious, spiritual and cultural reasons (table 1). Modern iterations of IF involve refraining from normal societal patterns of eating that follow the 24-hour period of light and day and restrict caloric intake to defined window periods.

These IF protocols can be divided into those that encourage time restricted eating
Key messages

What is already known?

- Intermittent fasting (IF) consists of alternating periods of voluntary fasting and unrestricted caloric consumption and is used in various religious, cultura and personal contexts.

- IF has been shown to facilitate weight-loss and improve glycaemic control among other metabolic processes.

- The exact mechanisms of how IF can improve health are unknown, however, theories derived largely from animal studies suggest increased ketosis, reduction in reactive oxidative species production and/or optimisation of central and peripheral circadian physiological processes.

What are the new findings?

- Alongside weight-loss, IF has been proposed to reduce systemic inflammation and may be of benefit in patients with certain chronic conditions associated with inflammation, such as metabolic syndrome.

- IF may have metabolic benefits resulting in reductions in weight and waist circumference that are independent of calorie restriction.

- The benefits of IF on body mass and cardiovascular risk factors may be further optimised with the addition of aerobic exercise.

- Modified alternate day fasting has been shown to reduce calorie intake on unrestricted caloric consumption days when individuals practice IF in real world conditions.

(TRE) patterns or alternate day fasting (ADF), with or without purposeful caloric restriction. TRE involves continuous short periods of IF, with food consumption typically within an 8 to 10 -hour window period, for example, the $16 / 8$ diet. $^{5}$ ADF comprises an alternating pattern between non-restricted days and fasted days with no caloric intake. ${ }^{5}$ However, in real world conditions these are poorly tolerated and have recently been replaced with modified ADF (mADF) protocols, which allow for an intake of up to $40 \%$ of the recommended daily caloric intake on fasted days. ${ }^{6}$

Many individuals who undertake IF report gaining psychological and spiritual health 
Table 1 Examples of fasting practices from ancient and modern societal practices

\begin{tabular}{|c|c|c|}
\hline Fasting practice & Pattern of fasting & Dietary restriction during fasting \\
\hline \multicolumn{3}{|l|}{ Religious practices } \\
\hline Ramadan (Islam) & $\begin{array}{l}29 \text { or } 30 \text { consecutive days of fasting during } \\
\text { daylight hours }\end{array}$ & No food or liquids consumed \\
\hline Yom-Kippur (Judaism) & 1 day of fasting for 24 hours & No food or liquids \\
\hline Proșadhopavāsa (Jainism) & Fasting on day 8 and 14 of the lunar cycle & $\begin{array}{l}\text { Abstinence from the four categories of } \\
\text { consumables: } \\
\text { 1. Food that satisfies entire hunger such as } \\
\text { rice, wheat, vegetables. } \\
\text { 2. Water. } \\
\text { 3. Sweets. } \\
\text { 4. Oral fresheners after meals. }\end{array}$ \\
\hline Lent (Christianity) & $\begin{array}{l}\text { Fasting for } 40 \text { days, fast during the day and } \\
\text { breaking fast at sunset }\end{array}$ & $\begin{array}{l}\text { Abstinence from meat, eggs, dairy products, } \\
\text { olive oil and alcohol }\end{array}$ \\
\hline Baguan zhai (Buddhism: eightfold fast) & $\begin{array}{l}\text { Do not eat after noon (midday), break-fast } \\
\text { the next morning }\end{array}$ & Abstinence from meat and fish \\
\hline $\begin{array}{l}\text { Alternate day fasting, for example, } 5: 2 \\
\text { protocol }\end{array}$ & Calorie restriction on 2 days of the week & $\begin{array}{l}\text { No particular dietary restriction but significant } \\
\text { calorie restriction }\end{array}$ \\
\hline $\begin{array}{l}\text { Modified alternate day fasting for example, } \\
\text { modified 5:2 protocol }\end{array}$ & $\begin{array}{l}\text { Intake of up to } 40 \% \text { of energy requirements } \\
\text { on } 2 \text { days of the week }\end{array}$ & $\begin{array}{l}\text { Up to } 40 \% \text { of recommended energy } \\
\text { requirements but no particular dietary } \\
\text { restriction }\end{array}$ \\
\hline
\end{tabular}

benefits from periods of abstinence from food. Recent data have shown that there may also be physiological benefits to fasting, with murine studies showing that it is associated with increased life expectancy; a reduction in the production of reactive oxygen species; improved inflammatory marker profiles (TNF- $\alpha$ and ceramides) and metabolic profiles (reduced body weight and fat mass). ${ }^{17}$ It is thought that IF may improve health by reducing systemic inflammation or by promoting autophagy (internal cellular degeneration and regeneration processes). ${ }^{189}$

\section{Exploring the mechanism behind IF}

TRE practices are increasing in popularity in the general population, with recent evidence supporting their use for diabetes and weight management in clinical populations under medical guidance. ${ }^{3}$ These regimes have been shown to be well-tolerated, and lead to an average of $20 \%$ unintentional reduction in caloric intake. ${ }^{10}$ It has been suggested that TRE may have additional benefits beyond calorie restriction, with several studies showing that these dietary patterns can improve insulin sensitivity and metabolic markers such as systolic blood pressure, $\mathrm{HbAlc}$, fat mass and triglycerides, even in eucaloric conditions. ${ }^{10}$

There are three main theories that have been suggested to explain this metabolic effect; (i) the Ketosis theory, (ii) oxidative stress hypothesis and (iii) the circadian rhythm hypothesis. The 'Ketosis theory' has the greatest traction both in mainstream and scientific communities. ${ }^{357}$ The theory postulates that IF leads to a reduction of total calorie intake, causing a metabolic shift which results in greater fat metabolism and reduced fat storage $^{5}$ (figure 1 ). Studies on IF have also been shown to enhance insulin sensitivity, increase high-density lipoprotein cholesterol and decrease levels of low-density lipoproteins. ${ }^{5}$

The longer-term benefits of IF have also been attributed to reduced systemic inflammation (oxidative stress theory). This theory suggests the decreased energy intake and production of reactive oxidative species by the mitochondria results in a diminished cellular inflammatory process. ${ }^{5}$ There are also additional circadian benefits on the timing of food intake, as the performance and function of key physiological processes (food absorption and metabolism) vary in keeping with our internal 24-hour circadian rhythm. These processes are highly sensitive to the time-of-day impact at both a central and peripheral cellular level and require distinct periods of fasting for optimal physiological function (circadian rhythm hypothesis) ${ }^{5}$ IF has been shown to increase the expression of a circadian rhythm regulating gene, NR1D1, which lowers fat accumulation in the liver and adipose tissues, as well as reducing inflammation and decreasing susceptibility to metabolic syndrome. ${ }^{1}$

Understanding these circadian effects in detail will provide us with practical recommendations of how best to implement IF. This may involve matching caloric intake to diurnal patterns of hormone secretion (eg, insulin secretion or sensitivity), or times of day when gut motility and absorption are optimised, or when higher proportions of energy are produced by carbohydrate metabolism. ${ }^{11}$ A practical example of this is abstaining 


\section{Intermittent fasting underlying mechanisms}

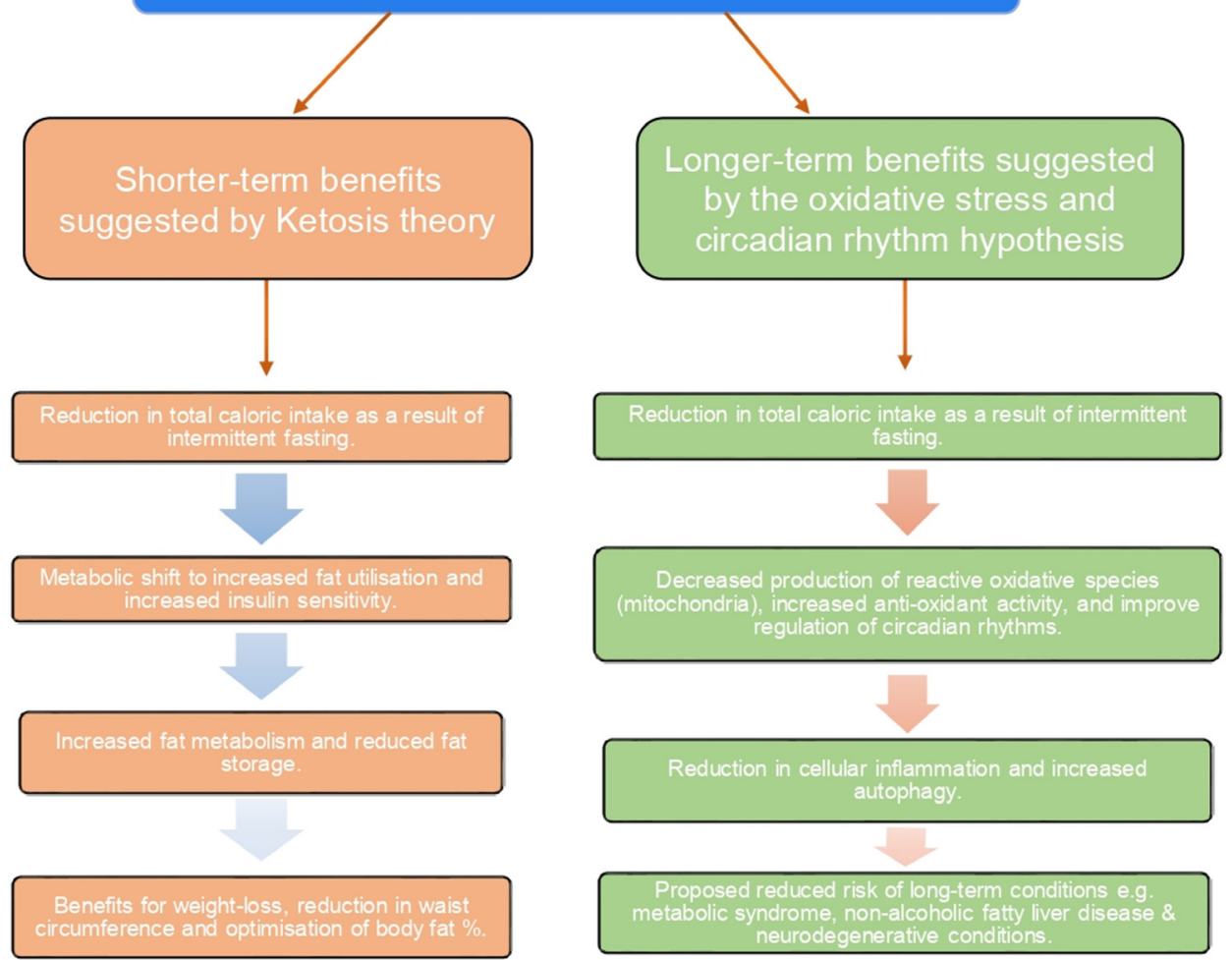

Figure 1 An overview of current theories on how intermittent fasting may impact health or performance.

from night-time eating, which decreases post-prandial glucose levels, increases insulin sensitivity and reduces the incidence of cardiovascular disease. ${ }^{5}$

\section{Benefits of fasting for metabolic health and beyond}

IF practices have demonstrated short-term positive impacts on patients' metabolic health, with a recent systematic review reporting significant reductions in weight (average body mass index decrease of $4.3 \%$ ) and waist circumference in overweight/obese patients. ${ }^{12}$ These findings were reproduced in both calorie restricted and eucaloric regimes, indicating a physiological benefit independent of reduced caloric intake. ${ }^{12}$ Thirty days of eucaloric IF in healthy volunteers has been associated with improved proteomic signatures and the upregulation of proteins involved in immune system regulation and DNA repair (short-term studies). ${ }^{1}$ It is hypothesised that this effect may improve individuals' health by reducing the risk of developing long-term conditions related to chronic inflammation. ${ }^{1}$

The interplay between IF and metabolic regulation may involve the circadian system. Recent studies on circadian rhythms have shown a diurnal variation of insulin and blood glucose levels. ${ }^{5}$ This raises the possibility of a time-of-day variation in the metabolic response to calorie consumption which is independent of the amount eaten. ${ }^{5}$ This hypothesis is supported by the finding of an increase in adiponectin (a key circadian system regulator linked to improved glycaemic control and insulin sensitivity) following TRE in physically active participants. ${ }^{10}$
This finding may also provide a practical example of the aforementioned 'circadian rhythm' hypothesis. ${ }^{5}$

It has been postulated that TRE, with its limited feeding windows, allows for the synchronisation of mealtimes to the circadian clock and hence optimises metabolic function. ${ }^{110}$ Interest into this circadian effect is growing, with recent studies exploring early TRE and delayed TRE. ${ }^{10}$ A greater understanding of the time-of-day impact of IF on metabolic function, and the influence of individual patients phenotype (ie, thinness), will allow us to implement these protocols better into personalised diets. ${ }^{13}$

For IF diets to be sustainable they must also be accompanied by continued lifestyle and behaviour change. mADF diets may provide this, with one study reporting a spontaneous $19 \%$ reduction in energy intake on unrestricted days in overweight patients completing the diet for weight loss. ${ }^{6}$ It is thought that IF patterns lead to a better awareness of eating habits and hunger, and improve eating behaviours on unrestricted days. ${ }^{6}$ The addition of tailored exercise programmes have also been shown to further enhance the health benefits of IF when taken with continued lifestyle changes. ${ }^{14}$

\section{IF for exercise performance}

In the era of personalised medicine, some athletes have seen IF as a way to optimise performance and maximise training benefits. Coordinating the timing of meals with athletes' circadian rhythms and training demands could be used to help maintain weight, improve sleep and aid recovery. This practice may be most applicable in short 
periodisation blocks, where nutritional strategies are used to aid specific training goals (ie, weight maintenance or aerobic capacity). ${ }^{2}$

Short-term eucaloric and hypocaloric TRE studies in physically active and resistance-trained adults, with adequate protein intake and hydration, have shown that IF practices are not detrimental to strength training adaptions such as muscle cross sectional area and fat free mass. ${ }^{248}$ Exercise in the fasted state has been shown to increase fat metabolism without impacting resting energy expenditure when compared with diets with continuous caloric intake throughout the day. ${ }^{28}$ There may also be additional benefits following short periods of TRE with improved immune system profiles and a reduction in IGF-1 production. ${ }^{15}$ Shortterm studies in soccer players taking part in 30 days of TRE (Ramadan) have also shown that periods of fasted exercise are well tolerated and did not increase the incidence of injury. ${ }^{1617}$

Despite these promising results from small scale, short-term studies there is currently a paucity of studies to demonstrate whether IF can reduce inflammation, injury, or illness risk in athletes in the long-term. Further research will be needed to establish the feasibility and benefit of various TRE protocols, that include overnight fasting and breakfast skipping, and further comparison of hypocaloric and eucaloric IF diets to generate optimal IF protocols for the athletes who wish to fast while training. ${ }^{5}$

\section{CONCLUSION}

IF practices are increasingly common in modern society and are well tolerated. Preliminary evidence supports their use in clinical populations at risk of metabolic conditions, and they may be of significant benefit to individuals by mechanisms which reduce systemic inflammation. Further research will allow us to understand the exact mechanisms behind the health benefits of IF and how these may be used in community populations to help fasting athletes train and prepare for competition.

\section{Twitter Irfan Ahmed @irfan_sem}

Contributors IA: devised the main conceptional ideas of the manuscript.IA, NS and SM: drafted the manuscript.IA, NS, SM, KC and SA: involved in structuring, researching and editing of the manuscript.I.A approved the final version of the manuscript.SM and NS contributed equally to this paper.

Funding The authors have not declared a specific grant for this research from any funding agency in the public, commercial or not-for-profit sectors.

Competing interests None declared.

Patient consent for publication Not applicable.

Ethics approval This study does not involve human participants.
Provenance and peer review Not commissioned; externally peer reviewed.

Open access This is an open access article distributed in accordance with the Creative Commons Attribution Non Commercial (CC BY-NC 4.0) license, which permits others to distribute, remix, adapt, build upon this work non-commercially, and license their derivative works on different terms, provided the original work is properly cited, appropriate credit is given, any changes made indicated, and the use is non-commercial. See: http://creativecommons.org/licenses/by-nc/4.0/.

\section{ORCID iDs}

Sumona Mandal http://orcid.org/0000-0002-6031-8091

Irfan Ahmed http://orcid.org/0000-0003-2146-4114

\section{REFERENCES}

1 Mindikoglu AL, Abdulsada MM, Jain A, et al. Intermittent fasting from dawn to sunset for 30 consecutive days is associated with anticancer proteomic signature and upregulates key regulatory proteins of glucose and lipid metabolism, circadian clock, DNA repair, cytoskeleton remodeling, immune system and cognitive function in healthy subjects. J Proteomics 2020;217:103645.

2 Correia JM, Santos I, Pezarat-Correia P, et al. Effects of intermittent fasting on specific exercise performance outcomes: a systematic review including meta-analysis. Nutrients 2020;12:1390.

3 Albosta M, Bakke J. Intermittent fasting: is there a role in the treatment of diabetes? A review of the literature and guide for primary care physicians. Clin Diabetes Endocrinol 2021;7:3.

4 Stratton MT, Tinsley GM, Alesi MG, et al. Four weeks of TimeRestricted feeding combined with resistance training does not differentially influence measures of body composition, muscle performance, resting energy expenditure, and blood biomarkers. Nutrients 2020;12:1126.

5 Dong TA, Sandesara PB, Dhindsa DS, et al. Intermittent fasting: a heart healthy dietary pattern? Am J Med 2020;133:901-7.

6 Harvey J, Howell A, Morris J, et al. Intermittent energy restriction for weight loss: spontaneous reduction of energy intake on unrestricted days. Food Sci Nutr 2018;6:674-80.

7 Stockman M-C, Thomas D, Burke J, et al. Intermittent fasting: is the wait worth the weight? Curr Obes Rep 2018;7:172-85.

8 Moro T, Tinsley G, Bianco A, et al. Effects of eight weeks of timerestricted feeding (16/8) on basal metabolism, maximal strength, body composition, inflammation, and cardiovascular risk factors in resistance-trained males. J Transl Med 2016;14:1-10.

9 Alirezaei M, Kemball CC, Flynn CT, et al. Short-term fasting induces profound neuronal autophagy. Autophagy 2010;6:702-10.

10 Réda A WM, Mériem M, Alexia P. Food timing, circadian rhythm and chrononutrition: a systematic review of time-restricted eating's effects on human health. Nutrients 2020;12:1-15.

11 Duboc H, Coffin B, Siproudhis L. Disruption of circadian rhythms and gut motility: an overview of underlying mechanisms and associated pathologies. J Clin Gastroenterol 2020;54:405-14.

12 Welton S, Minty R, O'Driscoll T, et al. Intermittent fasting and weight loss: systematic review. Can Fam Physician 2020;66:117-25.

13 Riveros-McKay F, Mistry V, Bounds R, et al. Genetic architecture of human thinness compared to severe obesity. PLoS Genet 2019;15:e1007603

14 Bhutani S, Klempel MC, Kroeger CM, et al. Alternate day fasting and endurance exercise combine to reduce body weight and favorably alter plasma lipids in obese humans. Obesity 2013;21:1370-9.

15 Moro T, Tinsley G, Longo G, et al. Time-restricted eating effects on performance, immune function, and body composition in elite cyclists: a randomized controlled trial. J Int Soc Sports Nutr 2020;17.

16 Chamari K, Haddad M, Wong DP, et al. Injury rates in professional soccer players during Ramadan. J Sports Sci 2012;30 Suppl 1:S93-102.

17 Eirale C, Tol JL, Smiley F, et al. Does Ramadan affect the risk of injury in professional football? Clin J Sport Med 2013;23:261-6. 